\title{
Temps, événements et mémoire médiatique : le cas du génocide des Arméniens dans la presse canadienne (1965-2005)
}

\begin{abstract}
Partant d'événements distincts dans le temps et l'espace, mais liés par essence, la mémoire historique se déploie, laissant apparaître au présent un passé aux contours incertains. À deux reprises, en 1982 et en 1985, l'ambassade de Turquie au Canada est le théâtre d'attentats : des " terroristes d'origine arménienne " signent des actes violents causant mort d'hommes, afin que la Turquie

Les auteurs : Joceline ChAвот est Professeure agrégée au Département d'histoire et de géographie de l'Université de Moncton.

Richard GoDIN est Chargé de cours à l'École des langues de l'Université Laval. reconnaisse sa responsabilité dans le génocide des Arméniens en 1915?.

Ces événements, qui relèvent alors de l'actualité, réactivent la construction mémorielle du génocide des Arméniens dans l'espace public. Les médias, la presse écrite en l'occurrence, pris entre les événements présents et passés, s'évertuent alors à faire état du génocide, de ses faits, de son oubli, de son déni par les autorités turques et une partie de la communauté internationale. Dès lors, la mémoire médiatique joue de l'histoire, épisodiquement, faisant passer de l'ombre à la lumière des faits classés en attente d'un jugement, si bien qu'elle participe parfois de la guerre des mémoires et des usages politiques du passé.
\end{abstract}

Dans une perspective mémorielle, il serait d'intérêt d'examiner le rôle tenu alors par les médias dans la relation des faits. À cet égard, l'épistémologie des sciences de l'information fixe des limites quant aux fonctions traditionnelles de la presse, lesquelles détermineront toujours la manière de relater les faits. D'où la pertinence d'examiner, d'un point de vue anthropologique, l'hypothèse voulant que la mémoire médiatique des événements se construise en fonction des postures journalistiques présentes dans la structure du discours : observation

'À compter des années 1970, un peu partout dans le monde, des attentats sont perpétrés par des groupes terroristes arméniens contre les membres des ambassades turques et leur famille. Par exemple, sont assassinés l'ambassadeur turc à Vienne, en 1975, l'épouse de l'ambassadeur à Madrid, en 1978, et le consul général à Los Angeles, en 1982. Gunther Michael M., Pursuing the Just Cause of their People. A Study of Contemporary Armenian Terrorism, Westport-New-York-Londres, Greenwood Press, 1986, $190 \mathrm{p}$ 
neutre, pacification des antagonismes, accusation quant à la position morale des bourreaux. En somme, cette triangulation aurait pour effet de façonner et de faire varier le récit dans le temps, et donc d'influencer les mémoires médiatique et historique.

Surgissent alors les questions de fond devant servir l'analyse. Comment les médias traitent-ils ces événements ? Comment la mémoire médiatique emprunte-t-elle à la mémoire historique et à la mémoire communautaire arménienne et turque dans l'interprétation de ces événements? Quelles transformations affectent le récit mémoriel ? Pour ce faire, nous analyserons un corpus d'articles parus dans trois journaux canadiens publiés à Montréal : La Presse, Le Devoir et The Gazette de 1965 à 2005. Cette périodisation tient compte des attentats des années 1980, mais aussi, plus largement, des commémorations décennales qui scandent la mémoire du génocide de 1915 et dont les manifestations publiques répercutées dans les médias alimentent les concurrences mémorielles. La composition du corpus sera précisée en cours d'analyse.

\section{Aux explications de la mémoire}

Aborder la construction mémorielle du génocide des Arméniens pour une période donnée et un espace déterminé suppose la mise en place d'un cadre théorique garantissant la validité de l'analyse. Puisqu'il servira à arpenter le corpus, le concept de mémoire collective doit être défini avec une justesse relative ${ }^{2}$ et s'inscrire dans une logique analytique. La foisonnante littérature portant sur l'étude de la mémoire sociale ne permet pas toujours d'en tracer des limites claires et précises. Pourtant, le champ de la connaissance visant la mémoire collective trouve un découpage temporel restreint (origines et contemporanéité) et épistémologique (sociologie, histoire, philosophie, anthropologie) $)^{3}$.

Reste néanmoins l'ancrage premier, qui relève de la sociologie durkheimienne et attribuable à Maurice Halbwachs, lequel nous permettra d'avancer dans ces "méandres " en gardant le cap. Parmi les réflexions de ce dernier, nous retenons cette affirmation : "Cependant c'est dans la société que, normalement, l'homme acquiert ses souve-

\footnotetext{
${ }^{2}$ Des études précédentes témoignent de cette nécessité de rigueur quant à l'opérationnalisation de ce concept. СНABOT Joceline et GODIN Richard, « Histoire, mémoire et médias : connaissance et reconnaissance du génocide arménien dans la presse québécoise, 1915-2005 ", in PÂQUET Martin (dir.), Faute et réparation au Canada et au Québec contemporains. Études historiques, Québec, Nota Bene, 2006, pp. 151-185. ${ }^{3}$ OLICK Jeffrey K., "Collective Memory ", in DaRITY William A. (dir.), International Encyclopedia of Social Sciences, $2^{\text {nd }}$ Edition, Detroit, Macmillan Reference, 2008, pp. 7-8.
} 
nirs, qu'il se les rappelle, et, comme on dit, qu'il les reconnaît et les localise $»^{4}$. Il faudrait aussi retenir l'idée selon laquelle la mémoire collective repose, entre autres, sur des faits sociaux colligés (archives, témoignages, vestiges, traditions, etc.) et accessibles au public dans une perspective relationnelle du présent vers le passé. S'entremêlent alors mémoires historique et médiatique dans le contexte politique et culturel de la société à l'étude.

De la mémoire historique, nous retiendrons l'image du couple histoire-mémoire, qui entretient des relations complexes, voire tumultueuses. En effet, du point de vue méthodologique, plusieurs éléments semblent les opposer : si la mémoire singularise le passé, l'histoire tend à la généralisation; la mémoire est nécessairement subjective, alors que l'histoire se veut objective. II s'agit là d'un couple bien mal assorti. Et pourtant, depuis une trentaine d'années, l'attention manifeste autour des pratiques commémoratives de toutes sortes, des questions du patrimoine matériel et immatériel, des musées à vocation identitaire et des monuments commémoratifs, témoigne d'un intérêt croissant pour la mémoire collective dans ses rapports problématiques au passé et à l'histoire ${ }^{5}$. D'aucuns expliquent cet intérêt d'abord par l'éloignement de la Deuxième Guerre mondiale et la disparition appréhendée de ses derniers témoins directs, mais aussi par les bouleversements consécutifs à l'effondrement de l'Union soviétique et la fin de la Guerre froide ${ }^{6}$.

Si la conjoncture historique et politique est importante, il est clair que les médias ont joué un rôle significatif en relayant au profit d'un large public les débats et les controverses des spécialistes entourant les événements douloureux d'un passé plus ou moins lointain : commémoration de la libération des camps nazis, guerre d'Algérie, reconnaissance du génocide des Arméniens ${ }^{7}$.

${ }^{4}$ HaLBWACHS Maurice, Les cadres sociaux de la mémoire, Paris, Félix Alcan, coll. «Les travaux de l'année sociologique ", 1925, p. 6.

${ }_{5}^{5}$ Parmi les nombreux ouvrages et articles traitant de cette question, signalons ceux de : CONNERTON Paul, How Societies Remember, Cambridge, Cambridge University Press, 1989, 121 p. ; LÉTOURNEAU Jocelyn et JeWsiewICKI Bogumil, "Politique de la mémoire », Politique et Sociétés, vol. 22, n², 2001, pp. 3-15; RIC氏UR Paul, La mémoire, l'histoire, l'oubli, Paris, Seuil, 2003, 720 p. et Rousso Henry, La Hantise du passé. Entretien avec Philippe Petit, Paris, Textuel, 1998, 143 p.

${ }^{6}$ Pomian Krzysztof, Sur l'histoire, Paris, Gallimard, coll. Folio histoire, 1999, 416 p. HARTOG François et Revel Jacques, "Note de conjoncture historiographique ", in Les Usages politiques du passé, Paris, EEHESS, 2001, pp. 13-24.

7 DOSSE, François, «L'histoire à l'épreuve de la guerre des mémoires », Cités, n³3, 2008, pp. 31-42; MASSERET Olivier, «La reconnaissance par le Parlement français du génocide arménien de 1915 ", Vingtième siècle. 
Cela étant, le couple turbulent histoire-mémoire est aussi travaillé par les liens indissociables qui font des jeux et des enjeux mémoriels un objet d'histoire. À ce titre, l'investigation historique de la mémoire collective permet l'exploration des diverses strates signifiantes de l'événement dont la clôture devient ainsi incertaine ${ }^{8}$. Cette présence du passé suscite un questionnement sur la temporalité événementielle, sa transmission, son emploi par la mémoire collective, son usage politique et son inscription dans l'espace public.

De la mémoire médiatique, nous retiendrons la mise en évidence récente de sa conception et de ses retombées heuristiques. Les drames collectifs mondiaux survenus à partir de la Deuxième Guerre mondiale jusqu'à aujourd'hui, conjugués avec le développement des médias modernes, contribuent à son usage dans ce champ d'études. Alors, il n'est plus saugrenu de juxtaposer les termes "mémoire » et "médiatique ", puisque l'événement rapporté devient inexorablement du contenu mémoriel, c'est-à-dire des archives.

Trois cas de figure précis tirés de la littérature illustrent cette assertion. Dans le premier cas, le concept est défini sans être nommé. Est évoquée alors la "mémoire publique " (proposition kantienne en opposition à mémoire collective), laquelle transcende la mémoire individuelle des membres de la société et renvoie aux "souvenirs" faisant l'objet de commémorations de masse: «le sens symbolique des événements retenus par la 'mémoire publique' dépend moins de l'expérience des contemporains que de son traitement par les médias $\Perp^{10}$.

Dans le deuxième cas, le concept prend forme, mais reste toutefois lié inextricablement à sa portion congrue, l'histoire, pour des raisons épistémologiques. II s'agit de la "mémoire historico-médiatique »: "La question de l'événement a été abordée par plusieurs disciplines, à commencer par l'histoire et la philosophie, mais aussi les sciences exactes et l'économie "n.

Dans le troisième cas, le concept se précise entièrement pour devenir «mémoire médiatique interdiscursive $~^{12}$, laquelle consiste en la transmission de connaissance des médias vers l'auditoire dans le but de constituer les mémoires individuelles et collectives. Dans tous

\footnotetext{
${ }^{8}$ RIC EUR Paul, «Événement et sens », Raisons pratiques. L'Événement en perspective, 1991, n² 2, pp. 51-55. - CHABOt Joceline et Godin Richard, "Histoire, mémoire et médias », op. cit., pp. 153-154.

10 BARASH Jeffrey A., «L'abîme de la mémoire. La mémoire collective entre expérience personnelle et identité politique ", Cités, n²9, 2007, p. 112 .

"CAlAbrese Steimberg Laura, La construction de la mémoire historico-médiatique à travers les désignations d'événements, Université Libre de Bruxelles, Travaux du CBL, 2006, disponible à l'adresse suivante http://webhol.ua.ac.be/, p. 4.

${ }^{12}$ MoIRAnd Sophie, Les discours de la presse quotidienne : observer, analyser, comprendre, Paris, Presses universitaires de France, 2007, $179 \mathrm{p}$.
} 
les cas, le but est de connoter la mémoire pour en tirer un effet, des représentations et des mythes politiques; l'acte est parfois involontaire, parfois volontaire, mais il en résulte deux dysfonctions à toute mémoire : oubli et déni.

\section{Analyse de la mémoire médiatique}

Les trois quotidiens canadiens à la base de l'analyse sont édités à Montréal. Leur importance relative dans l'espace public se traduit par des traditions respectives, les idées défendues en pages éditoriales et leurs tirages ${ }^{13}$. Fondé en 1884 , le journal La Presse se veut un rempart contre les détracteurs de l'unité canadienne et un défenseur de la langue française. Propriété de Gesca, une filiale de la multinationale Power Corp. of Canada, ce journal généraliste, avec des tirages moyens de 250.000 copies au cours de la période visée, concentre ses activités dans la grande région de Montréal, tout en maintenant quelques bureaux et correspondants en poste au pays et à l'international ${ }^{14}$.

Le Devoir est un quotidien indépendant depuis sa fondation, en 1910. À l'origine antibritannique et nationaliste canadienne, sa ligne éditoriale favorise la souveraineté du Québec depuis 1995. Son contenu économique, politique et culturel à valeur ajoutée vise traditionnellement l'élite intellectuelle québécoise et sa diffusion, bien que faible avec des tirages de 39.000 copies, se fait à la grandeur du Québec. Ce journal maintient en poste quelques correspondants et collaborateurs au pays et outre frontière, en plus d'une entente de réciprocité de services avec le quotidien français Le Monde ${ }^{15}$.

Le plus ancien des trois quotidiens, The Gazette, est probritannique à sa création, en 1785. Il est vu, encore aujourd'hui, comme le protecteur de l'héritage anglo-saxon au Québec. Ce quotidien de langue anglaise concentre ses activités et sa diffusion dans la grande région de Montréal là où se trouve la plus forte proportion d'anglophones résidant au Québec. Propriété de Postmedia Network Inc., son tirage se situe autour 150.000 copies $^{16}$.

\footnotetext{
${ }^{13}$ Les données exposées ici représentent les tirages moyens pour l'ensemble de la période 1965-2005: Centre d'études sur les médias, "Les quotidiens ont perdu pied "; Tirages des quotidiens québécois, 1998, disponibles à l'adresse suivante: http://www.cem.ulaval.ca/ et Newspapers Canada, Daily Newspaper Paid Circulation Levels: By Province, 2010, disponibles à l'adresse suivante $:$ http://www.newspaperscanada.ca/. ${ }^{14}$ Felteau Cyrille, Histoire de La Presse, Montréal, Les Éditions La Presse, 1983, 401 p.

${ }^{15}$ Comeau Robert et DesRochers Luc (dir.), Le Devoir : un journal indépendant (1910-1995), Québec, Presses de l'Université du Québec, 1996, 368 p. 
II est important de rappeler qu'entre 1965 et 2005, le Canada vit des relations tendues de nature identitaire avec le Québec. Dans ce contexte social et politique, la production journalistique vise naturellement à couvrir les événements s'y rapportant : la crise d'Octobre en 1970, l'élection d'un gouvernement indépendantiste au Québec en 1976 ou encore les référendums de 1980 et 1995. Bien que ces quotidiens couvrent largement cette réalité pancanadienne, des événements comme la commémoration du génocide des Arméniens ponctuent périodiquement l'actualité médiatique. C'est que la communauté arménienne canadienne, plus particulièrement québécoise, est bien intégrée à la société d'accueil et très active sur la scène publique et médiatique nationale. En effet, c'est à compter des années 1950 que le nombre d'Arméniens migrant au Canada augmente de façon significative. Ils proviennent de Grèce, d'Égypte et de Chypre. Le mouvement se poursuit dans les années 1960 et 1970 alors que les nouveaux arrivants viennent principalement du Liban et de la Syrie. Dans les années 1990, un nouveau contingent d'immigrants arméniens arrive d'Irak. En 2001, selon les données du recensement de Statistique Canada, la population d'origine arménienne établie à Montréal s'élève à près de 19.000 personnes. Mais, selon les Églises arméniennes de Montréal, la communauté compterait plutôt 35.000 membres $^{17}$. A la suite de ses recherches, l'anthropologue Annick Lenoir-Achdjian estime que ce dernier chiffre est beaucoup plus proche de la réalité ${ }^{18}$.

La communauté arménienne va rapidement se développer et se structurer autour d'organismes et d'institutions phares : églises, écoles et associations sportives et culturelles. Actuellement, on compte plusieurs dizaines d'organismes et d'associations ainsi que des journaux témoignant de la vitalité de cette communauté. Plusieurs de ces organismes sont étroitement liés à une organisation mère sise à l'extérieur du Canada, principalement aux États-Unis, mais aussi à des organisations situées ailleurs dans le monde. Cependant, près de la moitié d'entre eux sont indépendants et possèdent leur siège social à Montréal ${ }^{19}$.

Dans le cadre de son étude, Lenoir-Achdjian a montré que si des divisions politiques et idéologiques séparent les organisations communautaires arméniennes, celles-ci partagent toutefois un objectif

\footnotetext{
17 L'écart entre ces chiffres peut s'expliquer par le fait que certains membres de la communauté arménienne sont recensés à partir de leur lieu d'origine à savoir la Grèce, le Liban, etc. II peut aussi relever d'une surestimation de la population d'origine arménienne par les représentants de la communauté afin d'augmenter leur importance en tant que groupe. Tous ces chiffres sont donc à interpréter avec prudence.

${ }^{18}$ LENOIR-ACHDJIAN Annick, Appréhender la nation, vivre en diaspora: regards arméniens, thèse en anthropologie, Université de Montréal, 2001, pp. 128-129.

19 /bid., pp. 138-140.
} 
commun, soit le maintien et la promotion de la culture et de l'histoire arméniennes. Les travaux des chercheurs qui se sont intéressés à la question identitaire montrent que la transmission de la culture arménienne passe par celle de son histoire et de sa mémoire ${ }^{20}$. Au cœur de cette histoire et de cette mémoire, qui cimentent l'identité arménienne en diaspora, on retrouve l'événement traumatique qu'est le génocide de 1915. Dans Le Lien communautaire, Martine Hovanessian n'hésite pas à décrire cet événement comme le socle de la mémoire arménienne ${ }^{21}$. Pour la majorité des Arméniens de la diaspora, il est clair que la Turquie doit reconnaître et réparer les torts subis lors du génocide.

Au Québec, c'est dans les années 1970 et surtout 1980, alors que la communauté arménienne est en plein essor, que la demande de reconnaissance du caractère génocidaire des événements de 19151916 s'accentue. Certains des représentants les plus engagés dans la communauté n'hésitent pas à prendre publiquement la parole par le biais de lettres d'opinion qui paraissent dans les grands journaux québécois. Ainsi, dans un long article paru dans le quotidien Le Devoir, le 25 avril 1975, Vrej-Armen Artinian (membre de l'Association culturelle Hamazkaine et de l'administration de l'école arménienne SourpHagop), rappelle à ses concitoyens québécois que c'est pour lutter contre l'oubli et obtenir reconnaissance et réparation de la part de la Turquie que les Arméniens commémorent le $60^{\circ}$ anniversaire du génocide. Cette prise de parole par le biais de lettres et d'articles dans les journaux ainsi que les manifestations du 24 avril inscrivent la question de la reconnaissance du génocide dans l'espace public québécois. Dans les années 1990, la communauté formule de nouvelles revendications qui s'adressent non pas aux représentants des autorités turques, mais aux autorités canadiennes et québécoises. II s'agit alors de faire inscrire dans le calendrier civique montréalais et québécois le 24 avril journée commémorative du génocide arménien. C'est chose faite en avril 1999 au conseil municipal de Montréal, et en décembre 2003, à l'Assemblée nationale du Québec. À la suite d'une motion adoptée en 2004 par le Parlement canadien, le gouvernement du Canada reconnaît officiellement le génocide des Arméniens en 2006.

Nous n'avons pas dépouillé systématiquement les trois quotidiens retenus, c'est plutôt un choix raisonné qui a déterminé la constitution du corpus. L'analyse qui suit porte donc, d'une part, sur les anniver-

${ }^{20}$ Altounian Janine, La survivance. Traduire le trauma collectif, Paris, Dunod, 2000, 194 p. et HovanessIAN Martine, «Diasporas et identités collectives », Hommes et migrations. Diaspora arménienne et territorialités, n 1265, 2007, pp. 8-21.

${ }^{21}$ Hovanessian Martine, Le lien communautaire. Trois générations d'Arméniens, Paris, Armand Colin, 1992, $326 \mathrm{p}$. 
saires décennaux (1965, 1975, 1985, 1995, 2005), rappels répétés du génocide commis contre les Arméniens de l'Empire ottoman en 1915 ${ }^{22}$; et, d'autre part, sur des événements particuliers qui ont relancé sur la scène politique la question arménienne : les deux attentats terroristes commis par des ressortissants arméniens contre l'ambassade de Turquie à Ottawa, en 1982 et en 1985, et la polémique entourant la création, à Montréal, d'un monument commémoratif du génocide des Arméniens, entre 1996 et $1999^{23}$. Nous avons retenu tous les articles publiés autour des dates commémoratives et tous ceux parus à la suite de cette série d'événements. L'analyse de cette mémoire médiatique se décline en trois étapes : sa description; son traitement; ses modes de transformation.

\section{Description de la mémoire médiatique du génocide des Arméniens : les deux temps événementiels}

Une observation globale des éléments du corpus permet l'établissement d'un double constat quant à la transformation du récit médiatique des événements dans le temps. Ainsi, ce récit se déclinerait en deux temps événementiels distincts, comme nous le verrons un peu plus loin.

Premier constat : l'analyse montre (tableau 1) un nombre d'articles relativement faible pour les anniversaires décennaux, avec un sous-total de 39 textes, soit $27 \%$ du corpus; il en va autrement pour les périodes 1982-1985 et 1996-1999, avec la parution de 105 articles, soit près de 72,9\% du total des articles parus, une différence qui s'expliquerait par la nature des événements (actes terroristes et conflits entourant la création d'un monument commémoratif) et leur portée journalistique.

${ }^{22}$ C'est en 1965, soit lors de la commémoration du cinquantenaire des événements de 1915, que s'opère une prise de conscience dans l'ensemble de la communauté diasporique arménienne devant aboutir à la revendication de la reconnaissance du génocide par la Turquie et par la communauté internationale, voir ATTARIAN Varoujan, "La mémoire en diaspora. Actions pour la reconnaissance du génocide des Arméniens ». in L'Actualité du génocide des Arméniens, Actes du colloque organisé par le Comité de défense de la cause arménienne, Paris-Sorbonne 16-18 avril 1998, Paris, Édipol, 1999, pp. 261-267.

${ }^{23}$ La controverse politique entourant la construction à Montréal d'un monument à la mémoire des victimes du génocide des Arméniens a fait l'objet d'une étude spécifique dans un article récent: СНABOT Joceline, "Débats et controverses au sujet d'un monument commémoratif : la Réparation (Montréal, 1995-1999)". Questions de communication, série "Actes 5 ", Qualifier des lieux de détention et de massacre, Nancy, 2008, pp. 303-315. 
Tableau 1 : Nombre (n et \%) d'articles par quotidien

\begin{tabular}{|c|c|c|c|c|}
\hline $\begin{array}{c}\text { Quotidiens / } \\
\text { Périodes }\end{array}$ & La Presse & Le Devoir & The Gazette & Total \\
\hline 1965 & 1 & 3 & 1 & 5 \\
\hline 1975 & 0 & 2 & 1 & 3 \\
\hline 1985 & 3 & 1 & 3 & 7 \\
\hline 1995 & 5 & 3 & 6 & 14 \\
\hline 2005 & 2 & 2 & 6 & 10 \\
\hline S.-tot. & 11 & 11 & 17 & 39 \\
\hline$\%$ & 7,6 & 7,6 & 11,8 & 27 \\
\hline $1982-85$ & 7 & 11 & 39 & 57 \\
\hline $1996-99$ & 21 & 10 & 17 & 48 \\
\hline S.-tot. & 28 & 21 & 56 & 105 \\
\hline$\%$ & 19,4 & 14,6 & 38,9 & 72,9 \\
\hline Total & 39 & 32 & 73 & 100 \\
\hline$\%$ & 27 & 22 & 54 & \\
\hline
\end{tabular}

Deuxième constat : pour les deux périodes visées (1982-1985 et 1996-1999), le quotidien The Gazette fait paraître à lui seul 50 \% des articles, soit deux fois plus que chacun des quotidiens francophones. Cet écart s'observe autant pour la période des anniversaires décennaux, avec 11,8\% des articles comparativement à 7,6\% pour La Presse et Le Devoir, que pour la période relative aux événements entraînant des faits nouveaux, alors que The Gazette s'accaparera 38,9\% du corpus contre 19,4\% pour La Presse et 14,6\% pour Le Devoir. Une hypothèse s'impose quant à un tel écart entre le quotidien anglophone et les deux autres francophones: cela s'expliquerait par la nature des événements survenus (actes terroristes visant les membres du personnel de l'ambassade turque à Ottawa, en 1982 et en 1985) et le traitement qu'il en fera (exploitation du fait divers avec 31 articles). II faut aussi noter que La Presse couvrira de manière intensive les événements entourant l'érection à Montréal d'un monument commémorant les victimes du génocide des Arméniens de 1915, avec 21 articles pour la période 1996-1999. Rappelons que La Presse est un quotidien qui offre une place importante à l'actualité montréalaise.

À la lumière de ces deux constats, l'analyse offre de nouvelles perspectives, lesquelles correspondraient à deux temps événementiels (TE) distincts de la mémoire médiatique du génocide des Arméniens, c'est-à-dire : 1) les dates correspondant aux anniversaires décennaux, 
qui doivent être examinées en regard du caractère rituel de la commémoration à date fixe (le 24 avril) des victimes du génocide $\left(T_{1}\right)$; 2) les autres dates se rapportant à des événements nouveaux (TE2), lesquels surgissent de manière inattendue dans l'actualité, soit les attentats terroristes et l'érection controversée d'un monument commémoratif qui engagent une véritable guerre des mémoires entre les communautés arménienne et turque canadiennes. Dès lors, l'analyse propose une lecture en parallèle, et ce, toujours en proportion du corpus.

\section{Traitement de la mémoire médiatique : un schéma traditionnel}

La nature et le genre des textes offrent quelques indications pour ce qui est du traitement de la formation de la mémoire médiatique des événements. Dans l'ensemble, la nouvelle, qui relate les faits objectifs (observation neutre, par définition), sert de catalyseur et compte pour $54,6 \%$ du corpus, soit 7,6\% pour les anniversaires décennaux et $47 \%$ dans le cas des événements nouveaux (tableau 2).

Les représentations et les interprétations, qui découlent de la nouvelle et se traduisent par les différents autres genres disponibles, donnent une plus grande prégnance à cette mémoire en formation (pacification des antagonismes, accusations contre la position morale des bourreaux). Dans l'ensemble, les textes d'opinion occupent, à $25,2 \%$, une part importante du corpus, soit $8,4 \%$ en contexte de commémoration ( $\mathrm{TE}_{1}$ ) et $16,8 \%$ en situation de nouveauté (TE2).

Une remarque s'impose : si les autres genres (éditorial, analyse, reportage, autre) servent, d'une certaine façon, de fil d'Ariane au récit, ils créent, avec l'opinion, des espaces de discussion où se dévoilent les différences idéologiques et politiques opérant sur la mémoire des événements : demande de reconnaissance, par les uns; indifférence et déni, par les autres. Par exemple, dans une lettre au Devoir, en date du 25 avril 1975 (TE1), dont nous avons déjà cité un extrait, Vrej Armen Artinian interpelle ses concitoyens québécois et dégage la signification de la journée commémorative du 24 avril : «Pourquoi importuner nos concitoyens et nos amis [...] avec le souvenir terrible des atrocités commises à l'égard du peuple arménien ? [...] Les Arméniens ne peuvent oublier [...] tant que la question arménienne n'aura pas été résolue dans le sens de la justice et de l'équité ".

Trente ans plus tard, dans un entretien avec le journaliste Christian Rioux, l'historienne française, spécialiste du génocide des Arméniens, Claire Mouradian, fait écho aux propos d'Artinian : "Les Arméniens n'ont jamais oublié [...]. Ma grand-mère m'a toujours raconté ce qui s'était passé » (Le Devoir 23 avril 2005 / TE1). 
Tableau 2 : Distribution totale (n et \%) des genres pour l'ensemble du corpus selon les temps événementiels

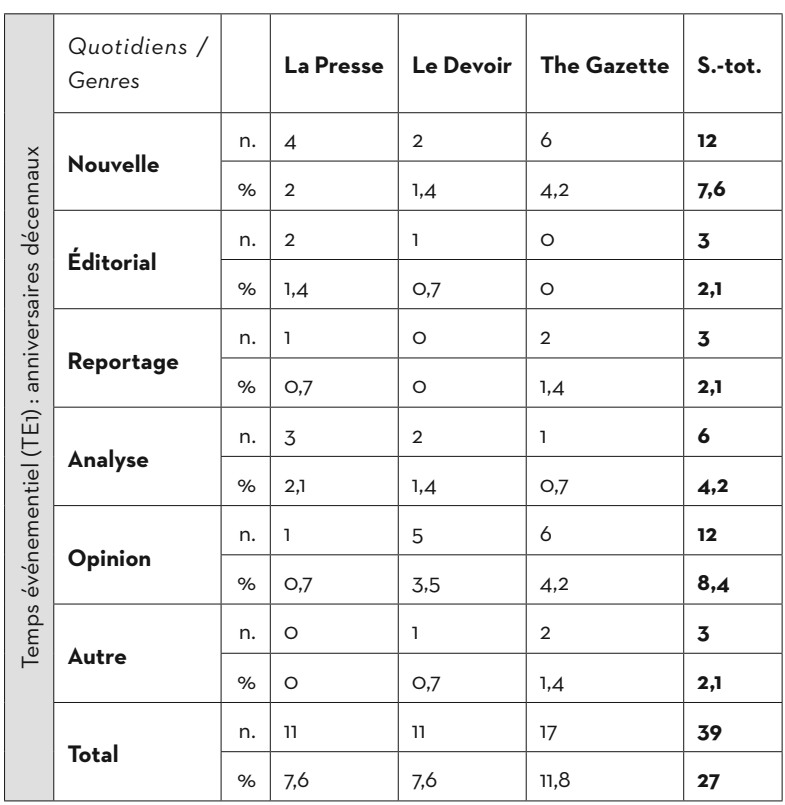

\begin{tabular}{|c|c|c|c|c|c|c|c|}
\hline \multirow{15}{*}{ 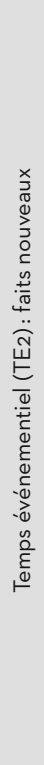 } & $\begin{array}{l}\text { Quotidiens / } \\
\text { Genres }\end{array}$ & & La Presse & Le Devoir & The Gazette & S.-tot. & Total \\
\hline & H & $\mathrm{n}$. & 16 & 13 & 39 & 68 & 80 \\
\hline & Nouvelle & $\%$ & 11 & 9 & 27 & 47 & 54,6 \\
\hline & r & $\mathrm{n}$. & 1 & 1 & 2 & 4 & 7 \\
\hline & Editorial & $\%$ & 0,7 & 0,7 & 1,4 & 2,8 & 4,9 \\
\hline & & $\mathrm{n}$. & o & 1 & 4 & 5 & 8 \\
\hline & Reportage & $\%$ & o & 0,7 & 2,8 & 3,5 & 5,6 \\
\hline & & $\mathrm{n}$. & 1 & o & 1 & 2 & 8 \\
\hline & Analyse & $\%$ & 0,7 & o & 0,7 & 1,4 & 5,6 \\
\hline & & $\mathrm{n}$. & 9 & 6 & 9 & 24 & 36 \\
\hline & Upinion & $\%$ & 6,3 & 4,2 & 6,3 & 16,8 & 25,2 \\
\hline & & n. & 1 & 0 & 1 & 2 & 5 \\
\hline & Autre & $\%$ & 0,7 & o & 0,7 & 1,4 & 3,5 \\
\hline & & $\mathrm{n}$. & 28 & 21 & 56 & 105 & 144 \\
\hline & lotal & $\%$ & 19,4 & 14,6 & 38,9 & 72,9 & 100 \\
\hline
\end{tabular}


Dans une représentation du passé contraire et irréconciliable avec celle des Arméniens, une lettre d'opinion signée Tolunay Yilmaz condamne les usages de la mémoire à des fins politiques par les terroristes arméniens qui ont perpétré des attentats: "The loss of Armenian lives was part of a global conflict that result in the death of more than 1,5 million people. 2,5 million Turks perished in that conflict. To bring back those sad memories in order to use them as tools of vengeance adds more shame to whatever happened more than 70 years ago " (The Gazette, 21 mars 1985). Quelques jours plus tard, Verge Tashian répond à ces propos en insistant sur les causes du terrorisme arménien : "The real problem is the first genocide of the century, that of the Armenian people, when in 1915, Enver and Talaat Pashas, heads of the then Turk government, ordered the planned and systematic massacre of my people " (The Gazette, 27 mars 1985). Dans le cadre éditorial, certains commentateurs n'hésitent pas à prendre position dans cette guerre mémorielle. Ainsi, Gilbert Grand, journaliste à La Presse, écrit : "Il aura [...] fallu l'assassinat de diplomates turcs en Europe et en Amérique du Nord par des terroristes se réclamant d'organisations nationalistes arméniennes pour soulever, au cours de la dernière décennie, la chape de plomb du silence jetée sur ce génocide refoulé » (La Presse, 3 août 1985 : A6).

En somme, un traitement médiatique de facture traditionnelle caractérise jusqu'ici le corpus : présentation des faits, leur analyse et la création d'espaces de discussion.

\section{Modes de transiormation de la mémoire médiatique : les valeurs neutre et non neutre}

La mémoire médiatique en transformation emprunte à la mémoire historique et à la mémoire communautaire arménienne et turque, et ce, selon le contexte de production : TEl, dans un contexte de production rituelle de la mémoire; $\mathrm{TE} 2$, dans un contexte de production médiatique stricto sensu et dont le résultat alimentera éventuellement la mémoire historique. En raffinant l'analyse par le regroupement des critères rédactionnels journalistiques sous deux grandes catégories de différenciation (neutre / non neutre), il appert que les temps événementiels (TE1-2) offrent des lectures mémorielles par nature dissemblables.

Ainsi, à la première catégorie de l'observation (neutre) correspond généralement la nouvelle, les faits bruts rapportés et non interprétés; à la seconde (non neutre), les représentations et les interprétations, correspondent l'éditorial, l'analyse, l'opinion et le reportage. Ces deux valeurs ne visent pas la mesure d'une charge émotive inhérente au 
récit mémoriel, mais plutôt à faire ressortir les voix multiples qui le composent et le transforment. II résulte de cette catégorisation une différence inversement proportionnelle entre les deux temps événementiels : TE1 porte une charge non neutre dans la récurrence des genres, avec un taux de 69,2\%, alors que TE2 présente du contenu à valeur neutre à raison de 64,8\% (tableau 3).

Tableau 3 : Proportion totale (\%) de la valeur différenciée des genres en fonction des temps événementiels (TE1-2)

\begin{tabular}{|c|c|c|}
\hline Charges / TE & TE1 & TE2 \\
\hline Neutre & 30,8 & 64,8 \\
\hline Non neutre & 69,2 & 35,2 \\
\hline Total & 100 & 100 \\
\hline
\end{tabular}

Toutefois, le récit mémoriel du génocide arménien, tel qu'il se déploie dans la presse canadienne, possède un certain nombre de topoï «neutre commun » à TE1 et TE2. Ainsi, il en va pour le décompte des victimes:

\footnotetext{
"Montreal's Armenian community Saturday observed the memory of more than 2,000,000 Armenians massacred in Turkey 50 years ago " (The Gazette, 26 avril 1965 / TE1).

«[...] environ 1 million et demi d'Arméniens ont été tués par ordre des Turcs » (La Presse, 23 avril 1995 / TE1).

"The Armenians blame Turkey for the 1915 massacre of more than one million of their countrymen »(The Gazette, 10 avril 1982 / TE2).

"It is believed as many as 1.5 million Armenians were executed or starved to death when they were expelled from Turkey in 1915 " (The Gazette, 30 août 1997 / TE2).
}

Par ailleurs, si la mémoire médiatique se construit sur la relation de faits nouveaux, elle se transforme aussi au gré des positions politiques prises par des individus, des groupes d'intérêt ou des membres des communautés visées. C'est ce qui distingue TE1 de TE2, soit la prédominance du discours public non neutre dans un récit mémoriel duquel s'élèvent des voix multiples. Ainsi, avant les années 1980, ce discours public se caractérise parfois par une certaine prudence quant à la qualification du crime. Par exemple: "En ce dimanche du souvenir, ils ont voulu rappeler leur douloureuse histoire [...] non pour raviver une haine, mais pour faire haïr son hideux visage " (Renaude Lapointe, La Presse, 27 avril 1965 / TE1). Soulignons que cette prudence dans le vocabulaire utilisé reflète la position du gouvernement fédéral canadien qui parle alors « d'événements tragiques " pour désigner le génocide des Arméniens.

Après 1980, les revendications formulées par les représentants 
de la communauté arménienne, mais aussi par d'autres acteurs de la scène publique et politique québécoise exigeant une reconnaissance officielle du génocide des Arméniens par les autorités politiques nationales et internationales transforment le discours public. À titre d'exemple, citons le cas d'Herbert Marx, député à l'Assemblée nationale du Québec, qui rédige un long article dans lequel il rappelle : «le peuple arménien est complètement frustré par la non-reconnaissance de son génocide [...]. Nul doute que le génocide arménien est gravé dans la mémoire individuelle et collective de tous les Arméniens. [...] il faut s'assurer que nous aussi, nous ne l'oublierons pas ", et il termine son texte par ce vœu : "Le génocide arménien est un fait historique [...]. II faut tout faire pour que des pays ainsi que des organismes internationaux reconnaissent ce fait " (Le Devoir, 23 mai 1984 : 9/TE1). L'année suivante, un éditorial de La Presse note la contribution unanime de l'Assemblée nationale du Québec à reconnaître le génocide des Arméniens (La Presse, 24 avril 1985 / TE1).

En 2004, le gouvernement du Canada fera de même. II faut noter que de ce discours émergent en échos les voix des antagonistes: revendications des uns, déni des autres. Par exemple : "That genocide took place is no longer a debate. It is an accepted fact, based on a mountain of factual evidence ", dira le président du Congrès des Canadiens arméniens, Taro Alepian (The Gazette, 10 avril 2005 / TE1). "Events were tragic - but not genocide", soutiendra pour sa part le consul général honoraire de Turquie en poste à Montréal, Gérard Battika, en parlant des événements de 1915 (The Gazette, 10 avril 2005 / TE1). Comme le souligne Jeffrey Barash, ce type de prise de parole qui transforme le récit interpelle une mémoire communautaire plus accessible à la remémoration directe et indirecte ${ }^{24}$.

\section{Conclusion}

Depuis plus de cinquante ans, la question de la reconnaissance du génocide des Arméniens est au cœur des manifestations mémorielles et identitaires des diasporas arméniennes au Canada, comme ailleurs dans le monde. La demande de reconnaissance du génocide de 1915 engage une guerre des mémoires qui oppose les communautés arménienne et turque dans une représentation du passé antagonique et irréconciliable obligeant les sociétés d'accueil à prendre position. Ces débats mémoriels, politiques et historiques ont été répercutés dans les médias canadiens tout au long de la période étudiée. Aujourd'hui, dans l'espace public médiatique, les trois plus importants quotidiens

${ }^{24}$ BARASH Jeffrey A., "L'abîme de la mémoire », op. cit., p. 111. 
du Québec privilégient une représentation du passé qui qualifie de génocide les événements de 1915. II faut souligner que depuis 1965 , Le Devoir qualifiait de génocide les massacres commis contre les Arméniens de l'Empire ottoman. En revanche, ce n'est que depuis le 6 juillet 2005, que le quotidien The Gazette reconnaît comme tel le génocide des Arméniens et autorise l'équipe éditoriale à ne plus utiliser de guillemets pour y référer ${ }^{25}$.

De cette analyse, nous constatons que le traitement des événements fait intervenir la mémoire médiatique TE1, laquelle opère dans la collectivité en la ritualisant, tout en forçant le débat public. Autrement, de manière sporadique, TE2 la fait intervenir en fonction d'événements inattendus qui peuvent survenir dans l'espace public, comme le montre l'histoire. Par exemple, les analyses réalisées sur le génocide rwandais ont bien mis en évidence que si les commémorations représentent pour les médias un moment privilégié pour rappeler au public ces événements tragiques à travers des scènes souvent chargées émotivement, elles évacuent trop rapidement la mise en contexte historique complexe qui entoure de tels drames historiques $^{26}$. Dans le cas de la Shoah, l'étude de David Cesarani portant sur la journée commémorative instituée en Grande-Bretagne en 2005, a révélé que les valeurs de l'information et les priorités médiatiques ont façonné cette commémoration. Comme dans le cas des trois journaux montréalais analysés dans cet article, la presse britannique a surtout été séduite par la controverse sur l'opportunité d'une journée commémorative consacrée au génocide nazi excluant d'autres génocides comme celui des Arméniens. Au terme de son analyse, l'auteur cherche à savoir si la mémoire de la Shoah sera désormais façonnée par les règles médiatiques ${ }^{27}$.

${ }^{25}$ Dans un communiqué en date du 6 juillet 2005, le Congrès des Canadiens arméniens fait état de la déclaration officielle de l'éditeur en chef du quotidien The Gazette quant à la prise de position sans équivoque du journal à l'égard du caractère génocidaire des massacres de 1915-1916. Il est aussi mentionné dans le communiqué que le quotidien emboîte ainsi le pas à d'autres grands quotidiens d'Amérique du Nord tels le Boston Globe et le New York Times. L'éditeur en chef de The Gazette déclare de la sorte : «It seems clear from the historical record that what took place in Turkey around 1915 amounted to a genocide, as defined in the 1948 UN convention on genocide [...]. As a result, reporters and editors are free to use the word genocide, without quotation marks, in relation to the Armenian tragedy and should avoid using qualifiers such as 'alleged', 'disputed' or 'what Armenians call'. We may report Turkish denials of such a claim when they are relevant, but we should not feel obliged to include such denials with every reference to the Armenian genocide ». Press Release from The Congress of Canadian Armenians / Congrès des Canadiens Arméniens, 2005, disponible à l'adresse suivante : http://www.crag.org.uk/.

${ }^{26}$ PAYETTE Dominique, "Les médias canadiens et le génocide rwandais. Une incompréhension lourde de sens ", Le Temps des médias, n 5, 2005, pp. 47-58 et VIDAL Claudine, "La commémoration du génocide au Rwanda : violence symbolique, mémorisation forcée et histoire officielle ", Cahiers d'études africaines, vol. XLIV (3), n 175, 2004, pp. 575-592. 
Cahiers

Mémoire et Politique

Si l'histoire cherche à mettre à distance le passé, à l'objectiver, la mémoire rend le passé présent, les souvenirs affectifs actuels. En empruntant à l'une et à l'autre, en résonnance avec l'actualité des événements et des commémorations, la mémoire médiatique sédimente les faits constitués, mais ouvre aussi à de nouvelles reconstructions du passé. Aujourd'hui, à la veille de la commémoration du centenaire du génocide des Arméniens, la guerre des mémoires entourant la qualification des événements est loin d'être terminée, mais, dans l'espace public canadien, le débat s'est apaisé. Quant aux médias, ils privilégient une ligne éditoriale commune reconnaissant le caractère génocidaire des crimes commis contre les Arméniens. Si ce discours public médiatisé permet de maintenir bien vivantes les traces mémorielles de cette tragédie humaine, on peut évidemment s'interroger sur sa capacité à offrir une compréhension fine d'un des premiers génocides du vingtième siècle. 